\title{
Asymptotic Connectivity in Wireless Networks Using Directional Antennas *
}

\author{
Pan Li, Chi Zhang, and Yuguang Fang \\ University of Florida, Gainesville, Florida 32611-6130 \\ \{lipanleo@, zhangchi@,fang@ece.\}ufl.edu
}

\begin{abstract}
Connectivity is a crucial issue in wireless networks. Gupta and Kumar show that with omnidirectional antennas, the critical transmission range for a wireless network to achieve asymptotic connectivity is $O\left(\sqrt{\frac{\log n}{n}}\right)$ if $n$ nodes are uniformly and independently distributed in a disk of unit area. In this paper, we investigate the connectivity problem when directional antennas are used. We find that there also exists a critical transmission range, which corresponds to a critical transmission power. We show that in the same propagation environment, when directional antennas use the optimal antenna pattern, the critical transmission power could be much smaller than that in networks using omnidirectional antennas. Moreover, to achieve asymptotic connectivity, it is known that each node has to have $O(\log n)$ neighbors when using omnidirectional antennas. We show that even using the transmission power level at which each node has only $O(1)$ neighbors when using omnidirectional antennas, we can still achieve the asymptotic connectivity with directional antennas.
\end{abstract}

\section{Introduction}

Omnidirectional antennas have been widely used in various kinds of wireless networks. But, due to the increased transmission range, the improved spatial reuse, and the decreased interference, directional antennas are potentially a better solution to the design of wireless networks. Although up to now, many research works on directional antennas focus on the design of MAC protocols ( [2] [8]), the network connectivity is indeed a critical issue.

Gupta and Kumar study the connectivity problem in [5] and show that there is a critical transmission range $O\left(\sqrt{\frac{\log n}{n}}\right)$ for the network to achieve asymptotic connectivity when $n$ nodes are uniformly and independently distributed in a disk of unit area. Some other papers like [4]

* This work was supported in part by the National Science Foundation under grant DBI-0529012 and under CAREER Award ANI-0093241.
[10] also investigate the connectivity issue. But all these works assume the use of omnidirectional antennas.

In the literature, there are also some works on connectivity with directional antennas [1] [3] [7]. However, these papers do not have a realistic directional antenna model. Neither do they take transmission and reception schemes into consideration, which are actually nonnegligible.

In this paper, we study the connectivity problem in wireless networks using directional antennas. Other than using the simple sector model, we introduce a more realistic directional antenna model, which consists of one main lobe with main lobe antenna gain $G_{m}$, as well as $N-1(N>1)$ side lobes with the same side lobe antenna gain $G_{s}$. We show that side lobe antenna gain has a significant impact on the network connectivity, which cannot be neglected. In our model, directional antennas can work either in the directional mode, or in the omnidirectional mode. So, according to the transmission and the reception schemes, we classify the networks using directional antennas into three categories: DTDR (Directional Transmission and Directional Reception) networks, DTOR (Directional Transmission and Omnidirectional Reception) networks, and OTDR (Omnidirectional Transmission and Directional Reception) networks.

Notice that for directional antennas, with the fixed transmission power, the directional transmission range is directly dependent on the omnidirectional transmission range given the directional antenna pattern $\left(G_{m}, G_{s}\right)$, the beam number $N$, and the path loss exponent $\alpha$. As a result, for each kind of networks, we derive the necessary and sufficient conditions on the omnidirectional transmission range for achieving asymptotic connectivity. We find that there also exists a critical omnidirectional transmission range. Thus, we can compare the power consumption when directional antennas are used to that when omnidirectional antennas are used, i.e., the power consumption in OTOR (Omnidirectional Transmission and Omnidirectional Reception) networks, by simply comparing their critical omnidirectional transmission ranges. For simplicity, we call the critical transmission range instead. We also call the corresponding transmission power the critical transmission power. 


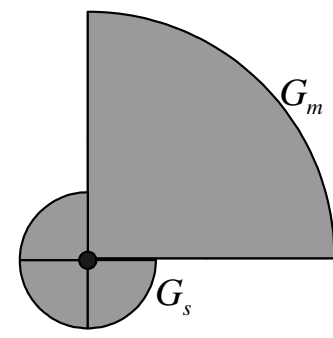

\section{Figure 1. Our switched beam directional an- tenna model.}

In order to minimize the critical transmission power when using directional antennas, for each of the three types of networks, we formulate a non-linear programming problem to find the optimum directional antenna pattern $\left(G_{m}, G_{s}\right)$, given the beam number $N$ and the path loss exponent $\alpha$. The impacts of the beam number $N$ as well as the path loss exponent $\alpha$ on the network connectivity are also analyzed.

We show that with the same beam number $N(N>2)$ and the same path loss exponent $\alpha(\alpha \in[2,5])$, the minimum critical transmission power in DTDR networks is smaller than that in DTOR and OTDR networks, which is further smaller than the critical transmission power in OTOR (Omnidirectional Transmission and Omnidirectional Reception) networks. But when the beam number $N$ is equal to 2, then with the same path loss exponent $\alpha$, the minimum critical transmission powers in DTDR, DTOR, and OTDR networks are all the same as that in OTOR networks.

Finally, it is known from [5] that for a wireless network using omnidirectional antennas to achieve connectivity, the transmission power should be set to the level at which each node has at least $O(\log n)$ neighbors, where $n$ is the total number of nodes in the network. In this paper, we show that even using the transmission power level at which each node has only $O(1)$ neighbors when using omnidirectional antennas, we can still achieve the asymptotic connectivity with directional antennas. This is true in all the three kinds of networks, i.e., DTDR, DTOR, and OTDR networks.

\section{Preliminaries}

We first introduce our directional antenna model. Denote a vector in three-dimensional space by $\vec{d}\left(\vec{d} \in R^{3}\right)$. Then the gain of an antenna in direction $\vec{d}$ is given by [9]:

$$
G(\vec{d})=\eta \cdot \frac{U(\vec{d})}{U_{\text {ave }}}
$$

where $U(\vec{d})$ is the power density in the direction $\vec{d}, U_{\text {ave }}$ is the average power density over all directions, and $\eta(0<$ $\eta \leq 1)$ is the efficiency of the antenna which accounts for losses. Clearly, we can see that an omnidirectional antenna has a gain of $0 \mathrm{~dB}$ and a directional antenna has a higher gain than that.

There are three primary types of directional antenna systems - switched beam antenna system, steered beam antenna system, and adaptive antenna system [6]. In this study, we use the switched beam antenna system, which consists of several highly directive, fixed, pre-defined beams and each transmission uses only one of the beams. Our study assumes that every antenna has $N(N>1)$ beams exclusively and collectively covering all directions. The main lobe antenna gain is denoted by $G_{m}$ and the side lobe antenna gain is denoted by $G_{s}$. We assume $G_{m}$ and $G_{s}$ are constants in the transmission direction and nontransmission directions, respectively. One such example with four beam directions is shown in Fig. 1. Moreover, directional antennas work either in the directional mode $\left(0 \leq G_{s}<1 \leq G_{m}\right)$ or in the omnidirectional mode $\left(G_{s}=G_{m}=1\right)$.

Let $P$ be the transmitting power, and $S$ the surface area of the sphere with center at the transmitter and radius $R$. As shown in Fig. 2, the surface area $A$ on the sphere for a beamwidth of $\theta$ is $2 \pi r h$, where $r$ is $R \sin \frac{\theta}{2}$, and $h$ is $R(1-$ $\left.\cos \frac{\theta}{2}\right)$. By the definition of antenna gain, when we neglect the side lobe gain $G_{s}$, we have [15]

$$
G_{m}=\frac{P / A}{P / S}=\frac{2}{\left(\sin \frac{\theta}{2}\right)\left(1-\cos \frac{\theta}{2}\right)},
$$

and when we consider the side lobe gain $G_{s}$, we have

$$
G_{m} \cdot U_{\text {ave }} \cdot A+G_{s} \cdot U_{\text {ave }} \cdot(S-A)=\eta \cdot P,
$$

where $G_{m}$ and $G_{s}$ are the main lobe directional antenna gain and the side lobe directional antenna gain, respectively. Since $P=S \cdot U_{\text {ave }}$, (1) can be simplified as

$$
G_{m} \cdot A+G_{s} \cdot(S-A)=\eta \cdot S,
$$

from which we can see that there is a relationship among the main lobe antenna gain $G_{m}$, the side lobe antenna gain $G_{s}$, and the beam direction number $N$, which is related to beam width $\theta$ by $N=2 \pi / \theta$.

We then introduce the power propagation model we use in this study. A general model [16] is given as follows:

$$
P_{r}(d)=P_{t} h\left(h_{t}, h_{r}, L, \lambda\right) \frac{G_{t} G_{r}}{d^{\alpha}},
$$

where $P_{t}$ and $P_{r}$ are the transmitted power and the received power, respectively, $G_{t}$ and $G_{r}$ are the gain factors for the transmitter antenna and the receiver antenna, respectively, $h_{t}$ and $h_{r}$ are the antenna heights of the transmitter and the receiver, respectively, $\mathrm{d}$ is the distance between transmitter 


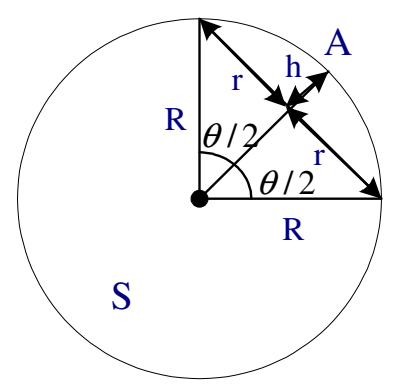

Figure 2. Illustration for calculating the main
lobe and the side lobe antenna gain.

and receiver, $L$ is the system loss factor not related to propagation $(L \geq 1), \lambda$ is the wavelength, $h(\cdot)$ is a function, and $\alpha$ is the path loss exponent.

Actually, the path loss exponent $\alpha$ characterizes the propagation environment and is usually ranged from 2 to 5 in outdoor environments [16].

\section{Necessary and sufficient conditions for asymptotic connectivity}

In this section we derive the necessary and sufficient conditions for achieving asymptotic connectivity in wireless networks using directional antennas. There are three categories of wireless networks in terms of the transmission and the reception schemes: Directional Transmission and Directional Reception (DTDR) networks, Directional Transmission and Omnidirectional Reception (DTOR) networks, and Omnidirectional Transmission and Directional Reception (OTDR) networks. We will discuss the connectivity problem in these three kinds of networks, respectively, in the following three subsections.

We first give the assumptions we use in this paper: (A1) There are $n$ static nodes uniformly and independently distributed in a disk of unit area on the plane.

(A2) All nodes are equipped with the same switched beam directional antennas. The number of beams is $N$. The main lobe and side lobe antenna gain are $G_{m}$ and $G_{s}$, respectively.

(A3) All nodes have the same transmission power and transmission range.

(A4) Each node randomly beamforms in one of the $N$ directions, with a probability of $\frac{1}{N}$.

(A5) Edge effects are neglected.

We denote this resulting graph by $G(V, E(g))$, where $V$ is the vertex set, and $E(g)$ is the edge set defined by a function $g: R^{2} \rightarrow[0,1]$. Notice that $g$ depends on the transmission and the reception schemes. So we use $g_{1}, g_{2}$, and $g_{3}$ in DTDR, DTOR, and OTDR networks, respectively.

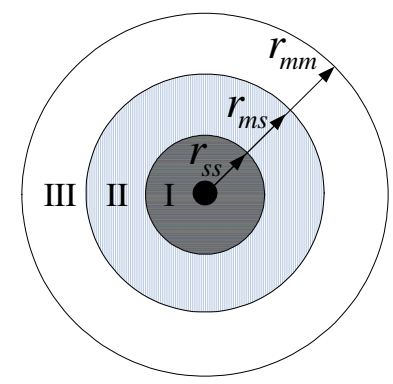

\section{Figure 3. The communication area of a node in DTDR networks}

\subsection{Directional transmission and direc- tional reception networks}

We first derive the necessary condition for achieving asymptotic connectivity in DTDR networks. Let $r_{0}$ denote the omnidirectional transmission range with the transmission power $P_{t}$. With the same transmission power, let $r_{m m}$ denote the transmission range when two nodes beamform to each other, $r_{m s}$ the transmission range when only one of the two nodes beamforms to the other, and $r_{s s}$ the transmission range when neither of the two nodes beamforms to the other one. Then, we obtain $r_{m m}=\left(G_{m} G_{m}\right)^{\frac{1}{\alpha}} r_{0}$, $r_{m s}=\left(G_{m} G_{s}\right)^{\frac{1}{\alpha}} r_{0}$, and $r_{s s}=\left(G_{s} G_{s}\right)^{\frac{1}{\alpha}} r_{0}$, where $\alpha$ is the path loss exponent.

Thus, the communication area of a node can be shown in Fig. 3. We can see that:

(1) Each node can communicate with the neighbors in Area I $\left(S_{1}^{D D}\right)$ with a probability of $p_{1}^{D D}$, where

$$
p_{1}^{D D}=1, \quad S_{1}^{D D}=\pi r_{s s}^{2} .
$$

(2) Each node can communicate with the neighbors in Area II $\left(S_{2}^{D D}\right)$ with a probability of $p_{2}^{D D}$, where

$$
\begin{aligned}
p_{2}^{D D} & =\frac{1}{N}+\frac{N-1}{N} \cdot \frac{1}{N}=\frac{2 N-1}{N^{2}}, \\
S_{2}^{D D} & =\pi\left(r_{m s}^{2}-r_{s s}^{2}\right) .
\end{aligned}
$$

(3) Each node can communicate with the neighbors in Area III $\left(S_{3}^{D D}\right)$ with a probability of $p_{3}^{D D}$, where

$$
p_{3}^{D D}=\frac{1}{N^{2}}, \quad S_{3}^{D D}=\pi\left(r_{m m}^{2}-r_{m s}^{2}\right) .
$$

(4) Each node cannot communicate with the neighbors in other areas.

Denote the probability that two nodes, $x_{i}$ and $x_{j}$, which also denote the positions of these two nodes in $R^{2}$, in DTDR networks are connected by $g_{1}(x)$, where $x=x_{i}-x_{j}$. Then,

$$
g_{1}(x)=\left\{\begin{array}{ll}
p_{1}^{D D} & \text { if }\|x\| \leq r_{s s} \\
p_{2}^{D D} & \text { if } r_{s s}<\|x\| \leq r_{m s} \\
p_{3}^{D D} & \text { if } r_{m s}<\|x\| \leq r_{m m} \\
0 & \text { if }\|x\|>r_{m m}
\end{array},\right.
$$


which is actually the function that determines the edge set in the graph $G\left(V, E\left(g_{1}\right)\right)$.

Thus, the effective area $S^{D D}$ of nodes in DTDR networks is

$$
\begin{aligned}
S^{D D} & =p_{1}^{D D} S_{1}^{D D}+p_{2}^{D D} S_{2}^{D D}+p_{3}^{D D} S_{3}^{D D} \\
& =\left[\frac{1}{N}\left(G_{m}\right)^{\frac{2}{\alpha}}+\frac{N-1}{N}\left(G_{s}\right)^{\frac{2}{\alpha}}\right]^{2} \pi r_{0}^{2} .
\end{aligned}
$$

Let $a_{1}=\left[\frac{1}{N}\left(G_{m}\right)^{\frac{2}{\alpha}}+\frac{N-1}{N}\left(G_{s}\right)^{\frac{2}{\alpha}}\right]^{2}$. Then, $S^{D D}=$ $a_{1} \pi r_{0}^{2}$.

To obtain our first main result, we need the following lemma [5].

Lemma 1 (i) For any $p \in[0,1]$,

$$
(1-p) \leq e^{-p}
$$

(ii) For any given $\theta \geq 1$, there exists $p_{0} \in[0,1]$, such that

$$
e^{-\theta p} \leq(1-p), \text { for all } 0 \leq p \leq p_{0} .
$$

(iii) For any fixed $\theta<1$ and for all sufficiently large $n$,

$$
n\left(1-\frac{\log n+c}{n}\right)^{n-1} \geq \theta e^{-c} .
$$

In the following, we use the notations $r_{0}(n), r_{m m}(n)$, $r_{m s}(n)$, and $r_{s s}(n)$ to replace $r_{0}, r_{m m}, r_{m s}$, and $r_{s s}$ respectively, indicating that these transmission ranges are dependent on the number of nodes $(n)$ in order to achieve connectivity.

Let $P^{(k)}\left(n, r_{0}(n)\right), k=1,2, \ldots$ denote the probability that the graph $G\left(V, E\left(g_{1}\right)\right)$ has at least one order-k component, i.e., a graph component which has $k$ nodes [17], and $P_{d}\left(n, r_{0}(n)\right)$ the probability that $G\left(V, E\left(g_{1}\right)\right)$ is disconnected. Then, we have the following theorem.

Theorem 1 (i) In DTDR networks, if $a_{1} \pi r_{0}(n)^{2}=$ $\frac{\log n+c(n)}{n}$, then

$$
\liminf _{n \rightarrow+\infty} P_{d}\left(n, r_{0}(n)\right) \geq e^{-c}\left(1-e^{-c}\right),
$$

where $c=\lim _{n \rightarrow+\infty} c(n)$.

(ii) In DTDR networks, if $a_{1} \pi r_{0}(n)^{2}=\frac{\log n+c(n)}{n}$ and $\lim \sup _{n \rightarrow+\infty} c(n)<+\infty$, then $G\left(V, E\left(g_{1}\right)\right)$ is asymptotically disconnected with positive probability.

Proof: (i) We first consider the case where $a_{1} \pi r_{0}^{2}(n)=$ $\frac{\log n+c}{n}$ for a fixed $c$. Then, the probability $P^{(1)}\left(n, r_{0}(n)\right)$ that $G\left(V, E\left(g_{1}\right)\right)$ has at least one order-1 component satis- fies

$$
\begin{aligned}
& P^{(1)}\left(n, r_{0}(n)\right) \\
\geq & \sum_{i=1}^{n} P\left(\left\{\mathrm{i} \text { is the only isolated node in } G\left(V, E\left(g_{1}\right)\right)\right\}\right) \\
\geq & \sum_{i=1}^{n}\left(P\left(\left\{\mathrm{i} \text { is an isolated node in } G\left(V, E\left(g_{1}\right)\right)\right\}\right)\right. \\
& \left.-\sum_{j \neq i} P\left(\left\{\mathrm{i} \text { and } \mathrm{j} \text { are isolated nodes in } G\left(V, E\left(g_{1}\right)\right)\right\}\right)\right) \\
\geq & \sum_{i=1}^{n} P\left(\left\{\mathrm{i} \text { is isolated in } G\left(V, E\left(g_{1}\right)\right)\right\}\right) \\
& -\sum_{i=1}^{n} \sum_{j \neq i} P\left(\left\{\mathrm{i} \text { and } \mathrm{j} \text { are isolated in } G\left(V, E\left(g_{1}\right)\right)\right\}\right)
\end{aligned}
$$

Since we neglect the edge effects, we have

$$
\begin{aligned}
& P\left(\left\{\mathrm{i} \text { is isolated in } G\left(V, E\left(g_{1}\right)\right)\right\}\right. \\
= & \left(1-a_{1} \pi r_{0}^{2}(n)\right)^{n-1} .
\end{aligned}
$$

To calculate $P\left(\left\{\mathrm{i}\right.\right.$ and $\mathrm{j}$ are isolated in $\left.\left.G\left(V, E\left(g_{1}\right)\right)\right\}\right)$, we should consider two cases: case 1, the effective areas of nodes $i$ and $j$ are disjoint; case 2, the effective areas of nodes $i$ and $j$ are overlapping. Denote the events of case 1 and case 2 by $C_{1}$ and $C_{2}$, respectively, and the event that $\mathrm{i}$ and $\mathrm{j}$ are isolated in $G\left(V, E\left(g_{1}\right)\right)$ by $E_{1}$. Then,

$$
\begin{aligned}
& P\left(E_{1} \mid C_{1}\right)=\left(1-2 a_{1} \pi r_{0}^{2}(n)\right)^{n-2}, \\
& P\left(E_{1} \mid C_{2}\right)=\left(1-\delta a_{1} \pi r_{0}^{2}(n)\right)^{n-2} .
\end{aligned}
$$

where $1 \leq \delta<2$. Thus,

$$
\begin{aligned}
P\left(E_{1}\right) \leq & 1 \cdot\left(1-2 a_{1} \pi r_{0}^{2}(n)\right)^{n-2} \\
& +3 \pi a_{1} r_{0}^{2}(n) \cdot\left(1-\delta a_{1} \pi r_{0}^{2}(n)\right)^{n-2} .
\end{aligned}
$$

Substituting (4) and (5) in (3), we obtain

$$
\begin{aligned}
& P^{(1)}\left(n, r_{0}(n)\right) \\
\geq & n\left(1-a_{1} \pi r_{0}^{2}(n)\right)^{n-1} \\
& -n(n-1) \cdot\left\{\left(1-2 a_{1} \pi r_{0}^{2}(n)\right)^{n-2}\right. \\
& \left.+3 \pi a_{1} r_{0}^{2}(n)\left(1-\delta a_{1} \pi r_{0}^{2}(n)\right)^{n-2}\right\} .
\end{aligned}
$$

Using Lemma 1, for $a_{1} \pi r_{0}^{2}(n)=\frac{\log n+c}{n}$, and for any fixed $\theta<1$ and $\epsilon>0$, we have

$$
\begin{aligned}
& P^{(1)}\left(n, r_{0}(n)\right) \\
\geq & \theta e^{-c}-n(n-1)\left(3 \pi r_{0}^{2}(n) e^{-\delta(n-2) a_{1} \pi r_{0}^{2}(n)}\right. \\
& \left.+e^{-2(n-2) a_{1} \pi r_{0}^{2}(n)}\right) \\
\geq & \theta e^{-c}-(1+\epsilon) e^{-2 c}
\end{aligned}
$$


for all $n>N(\epsilon, \theta, c)$. Since $P^{(1)}\left(n, r_{0}(n)\right) \leq$ $P_{d}\left(n, r_{0}(n)\right)$, then

$$
P_{d}\left(n, r_{0}(n)\right) \geq \theta e^{-c}-(1+\epsilon) e^{-2 c},
$$

for all $n>N(\epsilon, \theta, c)$.

In the case that $c$ is a function $c(n)$ with $\lim _{n \rightarrow+\infty} c(n)=\bar{c}$, the proof is similar to that in [5], by which we can show that

$$
\liminf _{n \rightarrow+\infty} P_{d}\left(n, r_{0}(n)\right) \geq \theta e^{-(\bar{c}+\epsilon)}-(1+\epsilon) e^{-2(\bar{c}+\epsilon)} .
$$

Since (6) and (7) hold for all $\epsilon>0$ and $\theta<1$, (i) follows. (ii) From (i) we can see that when $\lim \sup _{n} c(n)<+\infty$, $\liminf \operatorname{int}_{n \rightarrow+\infty} P_{d}\left(n, r_{0}(n)\right)>0$. This directly leads to (ii).

Next, we derive the sufficient condition for achieving asymptotic connectivity in DTDR networks. We use some results from continuum percolation by Penrose [13] [14]. Consider a graph where nodes are distributed as a homogeneous Poisson process in $R^{2}$ with intensity $\lambda$. Two nodes, $x_{i}$ and $x_{j}$, in the graph are connected with probability $g_{1}\left(x_{i}-x_{j}\right)$, as defined in (2). We denote this graph by $G^{\text {Poisson }}\left(V, E\left(g_{1}\right)\right)$, where $V$ is the vertex set, and $E\left(g_{1}\right)$ is the edge set defined by function $g_{1}$. In addition to that, we put a node at the origin $\mathbf{0}$. Then the resulting point process is a Poisson process "conditioned to have a point at $\mathbf{0}$, in the sense of Palm measures" [13]. We denote this new graph by $G^{\text {Poisson }}\left(V^{\prime}, E\left(g_{1}\right)\right)$, where $V^{\prime}=V \cup\{\mathbf{0}\}$.

We define the connected components of $G^{\text {Poisson }}\left(V^{\prime}, E\left(g_{1}\right)\right)$ as clusters. Let $p_{k}(\lambda, r(\lambda))$ denote the probability that the cluster containing the origin has $k$ nodes. The percolation probability, denoted by $p_{+\infty}(\lambda, r(\lambda))$, is the probability that $\mathbf{0}$ lies in an infinite cluster when $\lambda \rightarrow+\infty$, i.e.,

$$
p_{+\infty}(\lambda, r(\lambda))=1-\sum_{k=1}^{+\infty} p_{k}(\lambda, r(\lambda)) .
$$

Since the function $g_{1}(x)$ satisfies $g_{1}(x)=g_{1}(-x)$ for $x \in R^{2}$, and $0<\int_{R^{2}} g_{1}(x) d x<+\infty$, then we have the following two lemmas.

Lemma 2 (Theorem 3 in [13]): In the graph $G^{\text {Poisson }}\left(V^{\prime}, E\left(g_{1}\right)\right)$,

$$
\lim _{\lambda \rightarrow+\infty} \frac{\sum_{k=1}^{+\infty} p_{k}(\lambda, r(\lambda))}{p_{1}(\lambda, r(\lambda))}=1 .
$$

This means that as $\lambda \rightarrow+\infty$, almost surely the origin lies in either an infinite-order cluster or an order- 1 cluster (i.e., it is isolated).

Lemma 3 (Theorem 6.3 in [11]): When $\lambda \rightarrow+\infty$, there is at most one infinite cluster in $G^{\text {Poisson }}\left(V^{\prime}, E\left(g_{1}\right)\right)$.
Based on Lemma 2 and 3, the following lemma can be easily derived.

Lemma 4 As $\lambda \rightarrow+\infty$, the probability that the graph $G^{\text {Poisson }}\left(V^{\prime}, E\left(g_{1}\right)\right)$ is connected is asymptotically the same as the probability that the graph $G^{\text {Poisson }}\left(V^{\prime}, E\left(g_{1}\right)\right)$ has no isolated nodes, i.e.,

$$
\begin{aligned}
& \lim _{\lambda \rightarrow+\infty} \operatorname{Pr}\left[G^{\text {Poisson }}\left(V^{\prime}, E\left(g_{1}\right)\right) \text { is connected }\right] \\
= & \lim _{\lambda \rightarrow+\infty} \operatorname{Pr}\left[G^{\text {Poisson }}\left(V^{\prime}, E\left(g_{1}\right)\right) \text { has no isolated nodes }\right] .
\end{aligned}
$$

In [13], it has been shown that

$$
p_{1}(\lambda, r(\lambda))=\exp \left(-\lambda \int_{R^{2}} g_{1}(x) d x\right) .
$$

Let $\lambda=n$. If $a_{1} \pi r_{0}^{2}(n)=\frac{\log n+c(n)}{n}$, then we have $\int_{R^{2}} g_{1}(x) d x=a_{1} \pi r_{0}^{2}(n)=\frac{\log n+c(n)}{n}$. So, from (8), the probability of the origin to be isolated is:

$$
\begin{aligned}
p_{1}(\lambda, r(\lambda)) & =\exp \left(-n \int_{R^{2}} g_{1}(x) d x\right) \\
& =\frac{1}{n} e^{-c(n)}
\end{aligned}
$$

Let $E^{\prime}(G)$ be the expected number of order-1 cluster and $p^{\prime}(G)$ the probability that there is at least one order- 1 cluster in graph $G$. Then,

$$
\begin{aligned}
p^{\prime}\left(G^{\text {Poisson }}\left(V^{\prime}, E\left(g_{1}\right)\right)\right) & \leq E^{\prime}\left(G^{\text {Poisson }}\left(V^{\prime}, E\left(g_{1}\right)\right)\right) \\
& =n \cdot p_{1}(\lambda, r(\lambda)) \\
& =e^{-c(n)}
\end{aligned}
$$

So, if $\lim _{n \rightarrow+\infty} c(n)=+\infty$, then $p^{\prime}\left(G^{\text {Poisson }}\left(V^{\prime}, E\left(g_{1}\right)\right)\right) \rightarrow 0$ as $n \rightarrow+\infty$.

Thus, from Lemma 4 we can obtain

$$
\begin{aligned}
& \lim _{\lambda \rightarrow+\infty} \operatorname{Pr}\left[G^{\text {Poisson }}\left(V^{\prime}, E\left(g_{1}\right)\right) \text { is connected }\right] \\
= & 1-p^{\prime}\left(G^{\text {Poisson }}\left(V^{\prime}, E\left(g_{1}\right)\right)\right) \\
= & 1 .
\end{aligned}
$$

Since when the number of nodes $n$ is large, the difference between $G^{\text {Poisson }}\left(V^{\prime}, E\left(g_{1}\right)\right)$ and $G\left(V, E\left(g_{1}\right)\right)$ is negligible [12], then Theorem 2 directly follows.

Theorem 2 In DTDR networks, if $a_{1} \pi r_{0}^{2}(n)=\frac{\log n+c(n)}{n}$ and $c(n) \rightarrow+\infty$, then the graph $G\left(V, E\left(g_{1}\right)\right)$ is asymptotically connected with probability 1 .

Combining Theorem 1 and Theorem 2, we arrive at the following theorem.

Theorem 3 In DTDR networks, $G\left(V, E\left(g_{1}\right)\right)$, with $a_{1} \pi r_{0}^{2}(n)=\frac{\log n+c(n)}{n}$, is connected with probability 1 as $n \rightarrow+\infty$ if and only if $c(n) \rightarrow+\infty$. 


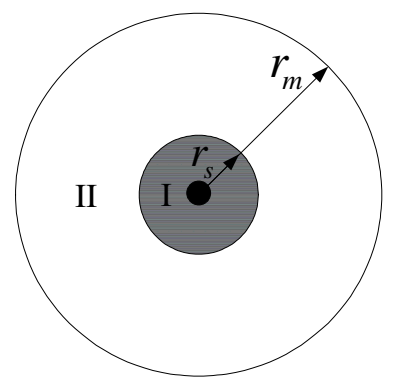

\section{Figure 4. The communication area of a node in DTOR networks.}

\subsection{Directional transmission and omnidi- rectional reception networks}

In this subsection, we derive the necessary and sufficient condition for achieving asymptotic connectivity in DTOR networks. Let $r_{m}$ and $r_{s}$ denote the transmission ranges, respectively, when the transmitter beamforms toward the receiver with main lobe gain $G_{m}$, and side lobe gain $G_{s}$. We can obtain:

$$
r_{m}=\left(G_{m}\right)^{\frac{1}{\alpha}} r_{0}, \quad r_{s}=\left(G_{s}\right)^{\frac{1}{\alpha}} r_{0} .
$$

where $\alpha$ is the path loss exponent.

As shown in Fig. 4, we observe:

(1) Each node can communicate with the nodes in Area I $\left(S_{1}^{D O}\right)$ with a probability of $p_{1}^{D O}$, where

$$
p_{1}^{D O}=1, \quad S_{1}^{D O}=\pi r_{s}^{2} .
$$

(2) Each node can communicate with the nodes in Area II $\left(S_{2}^{D O}\right)$ with a probability of $p_{2}^{D O}$. Recall that in Section 3.1, the communication is bidirectionally symmetric, i.e., if node $\mathrm{A}$ can communicate with node $\mathrm{B}$, then node $\mathrm{B}$ can also communicate with node A. However, in DTOR networks, the communication is bidirectionally asymmetric, i.e., if node A can communicate with node B, B may not necessarily be able to communicate with A. For example, if a node $\mathrm{B}$ is in Area II of node A, A is beamforming to B, but $\mathrm{B}$ is not beamforming toward $\mathrm{A}$, then $\mathrm{A}$ can send packets to $\mathrm{B}$ but $\mathrm{B}$ cannot send packets to A. We define that if two nodes cannot be connected in any direction, the connectivity level is 0 ; and that if two nodes can be connected only in one direction, the connectivity level is 0.5 ; and that if two nodes can be connected in both directions, the connectivity level is 1 . Thus we have

$$
\begin{aligned}
p_{2}^{D O} & =\frac{1}{N} \cdot \frac{1}{N} \cdot 1+2 \cdot \frac{1}{N} \cdot \frac{N-1}{N} \cdot \frac{1}{2} \\
& =\frac{1}{N}, \\
S_{2}^{D O} & =\pi\left(r_{m}^{2}-r_{s}^{2}\right) .
\end{aligned}
$$

(3) Each node cannot communicate with the neighbors in other areas.

Denote the probability that two nodes, $x_{i}$ and $x_{j}$, in DTOR networks are connected by $g_{2}(x)$, where $x=x_{i}-$ $x_{j}$. Then,

$$
g_{2}(x)=\left\{\begin{array}{ll}
p_{1}^{D O} & \text { if }\|x\| \leq r_{s} \\
p_{2}^{D O} & \text { if } r_{s}<\|x\| \leq r_{m} \\
0 & \text { if }\|x\|>r_{m}
\end{array} .\right.
$$

This is actually the function that determines the edge set in the graph $G\left(V, E\left(g_{2}\right)\right)$.

Thus, the effective area $S^{D O}$ of nodes in DTOR networks is

$$
\begin{aligned}
S^{D O} & =p_{1}^{D O} S_{1}^{D O}+p_{2}^{D O} S_{2}^{D O} \\
& =\left[\frac{1}{N}\left(G_{m}\right)^{\frac{2}{\alpha}}+\frac{N-1}{N}\left(G_{s}\right)^{\frac{2}{\alpha}}\right] \pi r_{0}^{2} .
\end{aligned}
$$

Let $a_{2}=\left[\frac{1}{N}\left(G_{m}\right)^{\frac{2}{\alpha}}+\frac{N-1}{N}\left(G_{s}\right)^{\frac{2}{\alpha}}\right]$. Then, $S^{D O}=$ $a_{2} \pi r_{0}^{2}$. Following the procedures in Section 3.1, we can get the following theorem:

Theorem 4 In DTOR networks, $G\left(V, E\left(g_{2}\right)\right)$, with $a_{2} \pi r_{0}^{2}(n)=\frac{\log n+c(n)}{n}$, is connected with probability 1 as $n \rightarrow+\infty$ if and only if $c(n) \rightarrow+\infty$.

\subsection{Omnidirectional transmission and di- rectional reception}

In OTDR networks, the connection function $g_{3}(x)$ is the same as the connection function $g_{2}(x)$ in DTOR networks. So the effective area of a node in OTDR networks is also the same as that in DTOR networks. Let $a_{3}=\left[\frac{1}{N}\left(G_{m}\right)^{\frac{2}{\alpha}}+\right.$ $\left.\frac{N-1}{N}\left(G_{s}\right)^{\frac{2}{\alpha}}\right]$, which is the same as $a_{2}$. Then we have:

Theorem 5 In OTDR networks, $G\left(V, E\left(g_{3}\right)\right)$, with $a_{3} \pi r_{0}^{2}(n)=\frac{\log n+c(n)}{n}$, is connected with probability 1 as $n \rightarrow+\infty$ if and only if $c(n) \rightarrow+\infty$.

\section{Minimizing the critical transmission power}

In [5], Gupta and Kumar show that when the number of nodes $n$ goes to infinity, the critical transmission range $r_{c}$ for OTOR (Omnidirectional Transmission and Omnidirectional Reception) networks to achieve connectivity is equal to $\sqrt{\frac{\log n+c(n)}{n \pi}}$, where $c(n) \rightarrow \infty$. Comparing Theorem 3, 4 , and 5, with their result, respectively, we have

$$
r_{c}^{i}=\frac{1}{\sqrt{a_{i}}} r_{c}, i=1,2,3 .
$$

where $\left\{r_{c}^{i}, i=1,2,3\right\}$ are the critical transmission ranges in DTDR, DTOR, and OTDR networks, respectively. 
Assume that the reception power should be larger than a threshold $P_{r}^{\text {thresh }}$ in order for the receiver to correctly receive the signal. Let $P_{t}, P_{t}^{1}, P_{t}^{2}$, and $P_{t}^{3}$ denote the critical transmission power in OTOR networks, DTDR networks, DTOR networks, and OTDR networks, respectively. Then,

$$
P_{t}^{i}=P_{t}\left(\frac{1}{a_{i}}\right)^{\alpha / 2},
$$

where $i \in\{1,2,3\}, \alpha \in[2,5]$ in outdoor environments.

So, in order to save power when we use directional antennas, our objective is to minimize $P_{t}^{i}$, respectively, for $i=1,2,3$. This is equivalent to maximize $\left(a_{i}\right)^{\alpha / 2}$, respectively, for $i=1,2,3$, as shown below:

$$
\begin{aligned}
& \operatorname{Maximize}\left\{\left(a_{i}\right)^{\alpha / 2}\right\} \\
& \text { subject to } \\
& \qquad 0 \leq G_{m} \cdot a+G_{s} \cdot(1-a) \leq 1 \\
& \quad G_{m} \geq 1,0 \leq G_{s} \leq 1
\end{aligned}
$$

where $a_{1}=\left[\frac{1}{N}\left(G_{m}\right)^{\frac{2}{\alpha}}+\frac{N-1}{N}\left(G_{s}\right)^{\frac{2}{\alpha}}\right]^{2}, a_{2}=a_{3}=$ $\left[\frac{1}{N}\left(G_{m}\right)^{\frac{2}{\alpha}}+\frac{N-1}{N}\left(G_{s}\right)^{\frac{2}{\alpha}}\right]$, and $a=\frac{1}{2}\left(\sin \frac{\pi}{N}\right)\left(1-\cos \frac{\pi}{N}\right)$.

Let $f\left(G_{m}, G_{s}, N, \alpha\right)=\frac{N-1}{N}\left(G_{s}\right)^{\frac{2}{\alpha}}+\frac{1}{N}\left(G_{m}\right)^{\frac{2}{\alpha}}$. Then, $\sqrt{a_{1}}=a_{2}=a_{3}=f\left(G_{m}, G_{s}, N, \alpha\right)$.

(1) when $N=2$, we have $a=\frac{1}{2}$ and $G_{m}+G_{s} \leq 2$.

For $\alpha=2$, it is obvious that the maximum values of $a_{1}$, $a_{2}$, and $a_{3}$ are all equal to 1 .

For $\alpha>2$, according to Holder's inequality, we obtain

$$
\left(G_{m}\right)^{\frac{2}{\alpha}}+\left(G_{s}\right)^{\frac{2}{\alpha}} \leq\left(G_{m}+G_{s}\right)^{\frac{2}{\alpha}} \cdot 2^{1-\frac{2}{\alpha}} \leq 2 .
$$

Thus, $a_{1} \leq 1, a_{2}=a_{3} \leq 1$.

(2) when $N>2$, since $\sqrt{a_{1}}=a_{2}=a_{3}=\frac{1}{N}\left(G_{m}\right)^{\frac{2}{\alpha}}+$ $\frac{N-1}{N}\left(G_{s}\right)^{\frac{2}{\alpha}}$, the three optimization problems presented in (9) can all be formulated as the same non-linear programming problem, as shown below:

$$
\begin{aligned}
& \text { Maximize }\left\{f\left(G_{m}, G_{s}, N, \alpha\right)\right\} \\
& \text { subject to } \\
& \qquad \cdot G \leq b \\
& A=\left(\begin{array}{cc}
a & 1-a \\
-a & -(1-a) \\
-1 & 0 \\
0 & -1 \\
0 & 1
\end{array}\right), b=\left(\begin{array}{c}
1 \\
0 \\
-1 \\
0 \\
1
\end{array}\right) \\
& G=\left(\begin{array}{c}
G_{m} \\
G_{s}
\end{array}\right) .
\end{aligned}
$$

Thus, for each $N>2$ and $\alpha \in[2,5]$, we can find optimum values of $G_{m}$ and $G_{s}$ to maximize $f\left(G_{m}, G_{s}, N, \alpha\right)$.

Denote the maximum value of $f\left(G_{m}, G_{s}, N, \alpha\right)$ by $\max _{\left\{G_{m}, G_{s}\right\}}(f)$. Fig. 5 shows the impacts of the path loss exponent on $\max _{\left\{G_{m}, G_{s}\right\}}(f)$ for $N \in[2,1000]$. We can
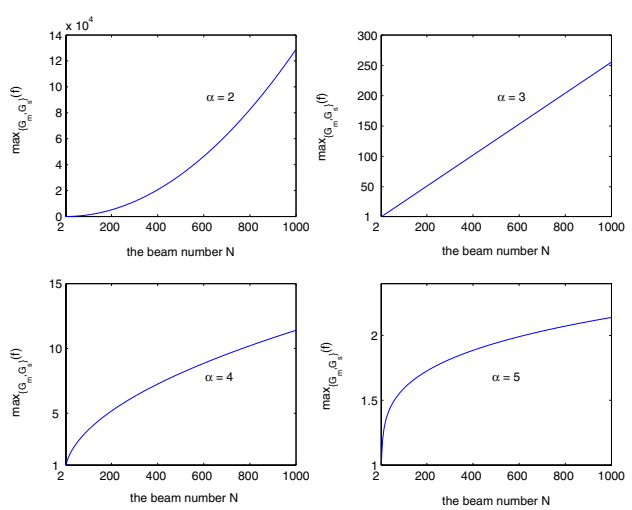

Figure 5. The impacts of path loss exponent on $\max _{\left\{G_{m}, G_{s}\right\}}(f)$.

see that with $\alpha$ fixed, $\max _{\left\{G_{m}, G_{s}\right\}}(f)$ increases as $N$ increases, while with $N$ fixed, $\max _{\left\{G_{m}, G_{s}\right\}}(f)$ decreases as $\alpha$ increases.

In conclusion, we find that when $N$ is equal to 2, $\max _{\left\{G_{m}, G_{s}\right\}}(f)=1$, and when $N$ is greater than 2, $\max _{\left\{G_{m}, G_{s}\right\}}(f)>1$, for all $\alpha \in\{2,3,4,5\}$.

Further more, for any $N>1$, we have

$$
\begin{aligned}
a_{1}-a_{2} & =a_{1}-a_{3} \\
& =f\left(G_{m}, G_{s}, N, \alpha\right) \cdot\left[f\left(G_{m}, G_{s}, N, \alpha\right)-1\right] .
\end{aligned}
$$

Thus, when the beam number $N$ is greater than 2 , the minimum critical transmission power in DTDR networks is smaller than that in DTOR and OTDR networks, which is further smaller than the critical transmission power in OTOR networks. But when the beam number $N$ is equal to 2 , the minimum critical transmission power in DTDR, DTOR, and OTDR networks are all the same as that in OTOR networks.

Moreover, since $f\left(G_{m}, G_{s}, N, \alpha\right)$ increases as either $G_{m}$ or $G_{s}$ increases, the maximum of $f(G m, G s, N, \alpha)$ can be achieved only when $G_{m} \cdot a+G_{s} \cdot(1-a)=1$, where $a=\frac{1}{2}\left(\sin \frac{\pi}{N}\right)\left(1-\cos \frac{\pi}{N}\right)$. Thus, $f\left(G_{m}, G_{s}, N, \alpha\right)$ can be represented by $f\left(G_{s}\right)$, where

$$
\begin{aligned}
f(G s)= & \left\{\frac{1}{N}\left[\frac{1}{a}-\left(\frac{1}{a}-1\right) G_{s}\right]^{\frac{2}{\alpha}}\right. \\
& \left.+\frac{N-1}{N}\left(G_{s}\right)^{\frac{2}{\alpha}}\right\} .
\end{aligned}
$$

(1) when $\alpha=2$, we have

$$
f(G s)=\frac{1}{a N}+\left(1-\frac{1}{a N}\right) G_{s} .
$$

As $N$ goes large, we obtain

$$
\begin{aligned}
1-\frac{1}{a N} & =1-\frac{2}{N\left(\sin \frac{\pi}{N}\right)\left(1-\cos \frac{\pi}{N}\right)} \\
& <1-\frac{4 N^{2}}{\pi^{3}}<0 .
\end{aligned}
$$




$$
\begin{aligned}
& \text { So, } \max _{\left\{G_{m}, G_{s}\right\}}(f)=\frac{1}{a N}>\frac{4 N^{2}}{\pi^{3}}, \text { and, } \\
& \max _{N}\left\{\max _{\left\{G_{m}, G_{s}\right\}}(f)\right\}=\lim _{N \rightarrow+\infty}\left\{\frac{1}{a N}\right\}=+\infty .
\end{aligned}
$$

(2) when $\alpha \in(2,5]$, with $N(N>1)$ and $\alpha$ fixed, $\max _{\left\{G_{m}, G_{s}\right\}}(f)$ is achieved when $\frac{\partial f(G s)}{\partial G_{s}}=0$. Let $G_{s}^{*}$ denote the result by solving the equation, then we obtain

$$
G_{s}^{*}=\frac{b}{a+(1-a) b},
$$

where $a=\frac{1}{2}\left(\sin \frac{\pi}{N}\right)\left(1-\cos \frac{\pi}{N}\right), b=\left[\frac{1-a}{a(N-1)}\right]^{\frac{\alpha}{2-\alpha}}$.

Substituting (11) into (10), we have $\max _{\left\{G_{m}, G_{s}\right\}}(f)=$ $f\left(G_{s}^{*}\right)$. We also find that for $N \in(1,+\infty), \alpha \in(2,5]$,

$$
\max _{N}\left\{\max _{\left\{G_{m}, G_{s}\right\}}(f)\right\}=\lim _{N \rightarrow+\infty}\left\{f\left(G_{s}^{*}\right)\right\}=+\infty .
$$

Since $\sqrt{a_{1}}=a_{2}=a_{3}=f\left(G_{m}, G_{s}, N, \alpha\right)$, then we obtain that for $i=1,2,3$, respectively,

$$
\max _{N}\left\{\max _{\left\{G_{m}, G_{s}\right\}}\left(a_{i}\right)\right\}=+\infty .
$$

From the above, we can see that for any $\alpha \in[2,5]$, $\left\{a_{i}, i=1,2,3\right\}$ can be made very large when the beam number $N$ goes large. Thus, for any arbitrarily large $n$, we can always find a parameter set $\left(N, G_{m}, G_{s}\right)$ so that $a_{i} \sim O(\log n)$.

Moreover, recall that when omnidirectional antennas are used, the expected number of neighbors of a node, which is $n \pi r_{0}^{2}(n)$, i.e., $\log n+c(n)$, has to approach infinity as $n \rightarrow+\infty$ in order to achieve connectivity. Define the critical number of neighbors of a node as $n \pi r_{0}^{2}(n)$ in DTDR, DTOR, OTDR, and OTOR networks. We conclude that if the critical number of neighbors of a node is only $O(1)$ by using omnidirectional antennas, we can still make the network connected by using directional antennas with the same transmission power.

\section{Conclusion}

In this paper we study the connectivity problem in wireless networks using directional antennas. We show that:

(1) When the beam number $N$ is 2 , the minimum critical transmission powers in DTDR, DTOR, and OTDR networks are all the same, which are equal to the critical transmission power in OTOR networks.

(2) When the beam number $N$ is larger than 2, the minimum critical transmission power in DTDR networks is smaller than that in DTOR, and OTDR networks, which is further smaller than that in OTOR networks.

(3) Moreover, when with some transmission power the critical number of neighbors of a node is only $O(1)$ by using omnidirectional antennas, we can still make the network connected by using directional antennas with the same transmission power.

\section{References}

[1] C. Bettstetter, C. Hartmann, and C. Moser. How does randomized beamforming improve the connectivity of ad hoc networks? In Proceedings of IEEE ICC, volume 5, Seoul, Korea, May 2005.

[2] R. R. Choudhury, X. Yang, N. Vaidya, and R. Ramanathan. Using directional antennas for medium access control in ad hoc networks. In Proceeding of ACM MOBICOM, Atlanta, Georgia, USA, September 2002.

[3] J. Diaz, V. Sanwalani, M. Serna, and P. Spirakis. Chromatic number of random scaled sector graphs. Theoretical Computer Science, 349(1):40-51, 2005.

[4] O. Dousse, F. Baccelli, and P. Thiran. Impact of interferences on connectivity in ad hoc networks. In Proceedings of IEEE Infocom, San Francisco, California, USA, April 2003.

[5] P. Gupta and P. Kumar. Critical power for asymptotic connectivity in wireless networks. Stochastic Analysis, Control, Optimization and Applications, A Volume in Honor of W.H. Fleming:547-566, 1998.

[6] R. Janaswamy. Radiowave Propagation and Smart Antennas for Wireless Communications. Kluwer Academic Publishers, Boston, USA, 2001.

[7] E. Kranakis, D. Krizanc, and E. Williams. Directional versus omnidirectional antennas for energy consumption and k-connectivity of networks of sensors. In Proceedings of OPODIS, Grenoble, France, December 2004.

[8] P. Li, H. Zhai, and Y. Fang. A directional mac protocol for ad hoc networks. In Proceedings of IEEE MILCOM, Washington, DC, USA, October 2006.

[9] J. Liberti and T. Rappaport. Smart Antennas for Wireless Communications. Prentice-Hall PTR, 1999.

[10] K. Madsen, F. Fitzek, G. Schulte, and R. Prasad. Connectivity probability of wireless ad hoc networks: Definition, evaluation, comparison. Special Issue of the International Journal on Wireless Personal Communications, 35(12):135-151, October 2005.

[11] R. Meester and R. Roy. Continuum Percolation. Cambridge University Press, Cambridge, UK, 1996.

[12] M. Penrose. Random Geometric Graphs. Oxford University Press, Oxford, UK, 2003.

[13] M. D. Penrose. On a continuum percolation model. Advances in Applied Probability, 23(3):536-556, 1991.

[14] M. D. Penrose. The longest edge of the random minimal spanning tree. The Annals of Applied Probability, 7(2):340 361, 1997.

[15] R. Ramanathan. On the performance of ad hoc networks with beamforming antennas. In Proceedings of ACM MobiHoc, Long Beach, California, USA, October 2001.

[16] T. Rappaport. Wireless Communications: Principles and Practice (Second Edition). Prentice-Hall PTR, 2002.

[17] W. Tutte. Graph Theory. Cambridge University Press, Cambridge, UK, 2001. 